\title{
Order and serial position effects in response time with multiple-item probe recognition
}

\author{
RAY PIKE, KEN JACKSON, and LEN DALGLEISH \\ University of Queensland, St. Lucia, Australia
}

\begin{abstract}
We examined the order effect in item-recognition response time, that is, differences in response time for multiple-item probes containing items in the same or in the reverse order as those in the memory set. Experiment 1 used the response condition in which only one item must be positive for a positive response, Experiment 2 used homogeneous probes in which all the items are either positive or negative, and Experiment 3 used the condition in which all the items must be positive. Of particular interest were the serial position variations in order effects for probes containing items that were adjacent in the memory set. We previously found that such effects are an indication of subjective grouping of the memory set and the matching of the probe with these subgroups. The order effect in the one-positive condition was only weak in most cases, but it was strong with homogeneous probes when the memory set was objectively grouped or was ungrouped but with a constant set size. There were also strong order effects in the all-positive condition for probes with items that were nonadjacent in the memory set. Our results are interpreted in terms of a parallel match process based on a distribution over position of items in subjective or objective groups. We account for the origin of the distribution-over-position process in terms of multiple representations of the grouped memory sets. The model assumes that each subgroup is represented in memory several, and perhaps very many, times and that considerable error in item positioning can occur over the multiple representations of any group.
\end{abstract}

In a recent series of experiments on item recognition, Pike, Dalgleish, and Jackson (1985) employed memory sets of four to six consonant items and probe sets of two or more items. They examined the effect on response latency of the correspondence between the order of items in the memory set and their order in the probe set. Only the individual items, not their order, were relevant to the response; all items in the probe had to be members of the memory set for a positive response. Strong order effects and serial position effects were found. The order effect, also demonstrated by Baumgarte and De Rosa (1973) with two-item probes, is that the average time to respond to those positive probes that contain items in the same order as in the memory set is less than the time to respond to positive probes with items in the reverse order. Apparently, incidental order information produces a marked effect on latency. We define serial position effects as variations in the order effect according to the position of items in the probe set relative to their position in the memory set.

Although order effects are not unexpected, they cannot easily be explained in terms of a theoretical model because of the presence of the serial position effects. Baumgarte and De Rosa (1973) came to few theoretical conclusions, except to point out that their data were difficult to explain by a serial search model. Pike et al. (1985) suggested that the order and serial position effects were caused by subjective grouping of the memory set into subsets. Pike et al. assumed the matching process to

Please address correspondence to Ray Pike, Department of Psychology, University of Queensland, St. Lucia, Queensland, Australia 4067. be holistic, or configurational, so that positive response strength is largely gained from items matched in identical positions in the subjective group and probe. For example, consider the six-item memory set ABCDEF, for which there may be subjective grouping into $A B C$ and $D E F$. Then consider the serial position effects for the same-order and reverse-order three-item probes $\mathrm{ABC}, \mathrm{CBA}$; BCD,DCB; CDE,EDC; DEF,FED. There should be strong order effects for the first and last of these pairs due to the presence in memory of representations derived from the subjective groups $A B C$ and DEF. Note that the probe pairs $B C D, D C B$ and $C D E, E D C$ may have reverse-order effects since the reverse-order probe has one item in the correct position. Thus it is the order of items within the subjective groups and not between them that is related to the order effect. In general, subjective grouping, in our study, is indicated when there are order effects for within-group probes (ABC, CBA, DEF, FED) but not for between-group probes (BCD,DCB; CDE,EDC). In our experiments, we used the all-positive response rule, so that the subjects were to say "yes" only if all items in the probe were positive, and positive probes always contained items that were adjacent in the memory set.

A complete development of the theoretical process by which the order and serial position effects are generated is discussed later. Before that discussion, we require data on the extent of the order effect with (a) different response conditions, (b) different memory set sizes and (c) probes containing items that are not adjacent in the memory set. The first of these requirements is to determine the generality of the order effect and the conditions under which holistic processing is or is not likely to occur. The sec- 
ond is to allow a greater variety of serial position effects, which together with (c) is necessary to make more extensive tests of theoretical models. Experiments 1 and 2 were concerned with (a), Experiment 2 was also concerned with (b), and Experiment 3 was concerned with (c).

Experiments 1 and 2 allowed us to further investigate the nature of subjective grouping and when it is likely to occur. We predicted that if conditions were such that single-item comparisons would be more likely, then the order effect would decrease. This is because subjects may make only single-item comparisons when the correspondence of the order of items in the memory and the probe sets is of no relevance to the response, as in the case for the response conditions used in Experiments 1 and 2. Thus little order effect was predicted because of the lack of holistic comparisons of probes with subjective groups. The procedure used in the first two experiments was similar to that used by Walker (1972), who also found strong evidence for subjective grouping, and that used by Pike et al. (1985), insofar as the memory sets were grouped into twoand three-item subgroups or they were ungrouped.

\section{EXPERIMENT 1}

In Experiment 1 we used the one-positive response condition, in which only one item in the probe must be positive for a positive response. Experiment 3 of Pike et al. (1985) demonstrated that subjects in the multiple-item probe task were considerably influenced by actual grouping in the memory set in the all-positive response condition. In addition, when an ungrouped memory set consisted of six items, subjects were likely to group items into two subsets of three items. We tested in Experiment 1 whether the same result would occur when using the onepositive condition, in which attention to groups of items seems less likely to occur. As in Pike et al.'s study, subjects underwent different sessions in which the memory set either was grouped in a particular way or was ungrouped, with presentation order randomized. That is, we induced a grouping strategy by employing grouped memory sets, the object being to see how such a strategy influences responding in the ungrouped condition and the relationship to the order effect.

\section{Method}

Subjects. Six undergraduate subjects were paid at a standard rate.

Stimuli and Apparatus. Each memory set consisted of 6 items drawn at random from the 21 consonants. Positive probes of size 3 contained three, two, or only one positive item in equal proportions, and negative probes contained all negative items. Positive and negative probes differed in a similar manner for probes of size 2 . Items in positive probes that contained at least two positive items were either in the same or in the reverse order in relation to items in the memory set. Positive and negative probes were equally likely. Memory sets were grouped into two subsets of three items, were grouped into three subsets of two items, or were ungrouped. If grouped, the space between subsets was equal to the space for a single item. Thus there were six experimental conditions, with two factors of probe size and grouping condition. Probe items were selected to correspond equally often to the different memory set serial positions, but were always adjacent in the memory set. The stimuli were $1 \times 1 \mathrm{~cm}$ in size, generated by a PDP-11/34 computer, and displayed simultaneously in the center of a Burroughs Self-Scan Display Panel at a distance of about $60 \mathrm{~cm}$ from the subject. The timing of the display and recording of the reaction time and response was controlled by a microcomputer in communication with the PDP-11/34.

Procedure. Each trial consisted of a 1.5-sec warning period during which asterisks were displayed. This was followed by the six-item memory set for $3 \mathrm{sec}$. After a delay of $1.5 \mathrm{sec}$, the probe set was displayed and was response terminated, or it was stopped after $3 \mathrm{sec}$ if there was no response. The one-positive decision rule was used (i.e., at least one probe item, at any position, must be positive), and each subject completed seven sessions. The first session was practice and contained six blocks of 50 trials, with each block corresponding to one of the probe set size $x$ memory set size $X$ memory set grouping combinations. There was then one session for each of the six conditions, and each session consisted of 10 practice trials followed by five blocks of 60 trials each. The assignment of subjects to the sequence of conditions was by means of a $6 \times 6$ Latin square so that some subjects experienced an ungrouped condition first.

\section{Results and Discussion}

We are mainly concerned with correct positive response times (RTs) for probes containing all positive items. Order and serial position effects were found for probes of size 2 but not for those of size 3 . For probe set size 2 , an analysis of variance (ANOVA) of mean response latencies produced a significant order effect $[F(1,5)=15.1, M S e$ $=4,011, p<.01]$ and an order $\times$ serial position interaction $[F(4,20)=4.7, M S e=1,560, p<.01]$. The grouping condition also interacted with serial position $[F(8,40)=2.46, M S e=9,544, p<.05]$. The overall extent of the order effect was $80 \mathrm{msec}$ (same-order mean $\mathrm{RT}=1,137 \mathrm{msec}$; reverse-order mean $\mathrm{RT}=$ $1,217 \mathrm{msec}$ ). There were no significant ANOVA effects for probe set size 3 , the order effect being only $48 \mathrm{msec}$ (same-order mean RT $=1,226 \mathrm{msec}$; reverse-order mean $\mathrm{RT}=1,274 \mathrm{msec}$ ). Error rates for probe sizes 2 and 3 were $5.6 \%$ and $4.8 \%$, respectively, for same-order probes and $7.2 \%$ and $5.3 \%$, respectively, for reverse-order probes. The overall mean RT for correct negative probes was $1,580 \mathrm{msec}$, with an error rate of $2.2 \%$.

Figures 1 and 2 present a comparison of the data of Pike et al. (1985) and the present data, using only probes with all items positive. There were no interactions with subjects so that the 2 subjects who were first presented with ungrouped memory sets showed serial position effects similar to those of the other 4 subjects. This is probably because the initial practice session utilized all conditions.

For probe set size 2 the general pattern of serial position effects is similar to that in Experiment 3 of Pike et al. (1985), although the extent is diminished (compare Figures 1 and 2). For probe set size 3 the effects are virtually nonexistent. It appears that, as predicted by the grouping hypothesis, the order effects are strongly related to the serial position effects. That is, if order effects arise from holistic comparisons of probes with subjective groups and with consequent serial position effects, then the less the occurrence of such comparisons, the weaker 


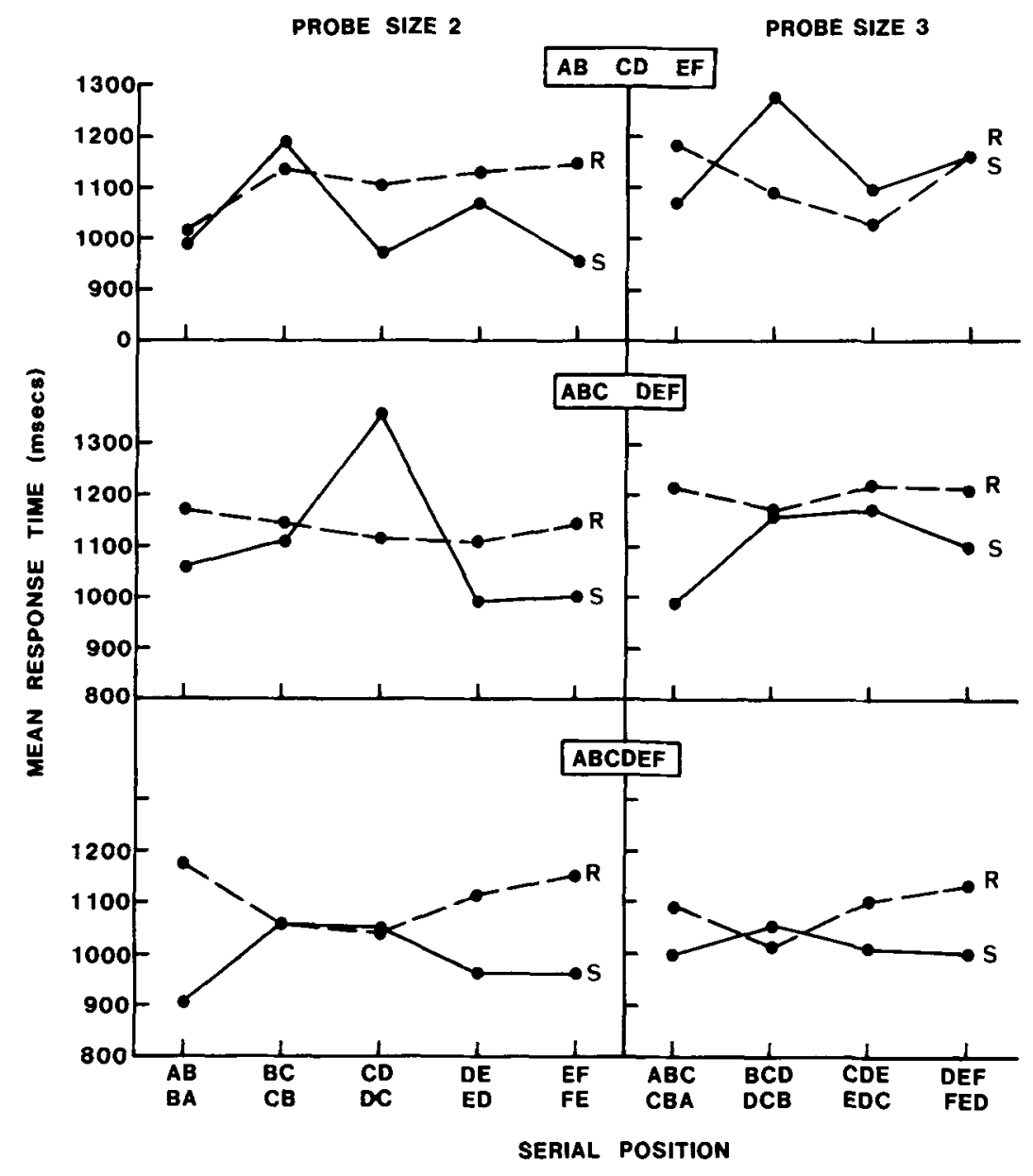

Figure 1. Mean latencies for the six conditions of Experiment 1. Memory set groupings appear in the boxes. $\mathbf{S}$ denotes same-order probes; $\mathbf{R}$ denotes reverse-order probes.

the serial position effects. Apparently, in the one-positive condition, subjects give less attention to grouping of items, so that single-item processing is dominant and the influence of subjecive grouping is only weak.

There are at least two interpretations of these data. One is to maintain that subjective grouping occurs only on some trials and the single-item comparisons are made on the remainder. Another interpretation is that both processes, comparisons of the probe with subjective groups and comparisons of single-probe items with memory items, occur in parallel. In both cases, we maintain that the order and serial position effects are caused by holistic matching of probes with subjective groups. The data do not support a serial scan with single items, so that the order effect is brought about by subjects taking the first item in the probe to perform the serial scan. In that case, the order effects should increase considerably from probe size 2 to probe size 3 , and this result is not found in this experiment or in Pike et al.'s (1985) study. The mean latencies are the same as or less than those in Pike et al. (1985), and they are similar for the two probe conditions. This may indicate that single-item matching is, on the average, no faster than holistic matching.
Because the order and serial position effects were only moderate in these data, we made an additional test of the one-positive condition. The subjects were presented only with ungrouped memory sets. Similar results were obtained, with order effects of $50 \mathrm{msec}$ and $61 \mathrm{msec}$ for probe sizes 2 and 3, respectively, and there were only weak serial position effects. Again, there was no significant increase in response time for the two probe set size conditions.

\section{EXPERIMENT 2}

We next used a different form of response condition, in which the probes were homogeneous with regard to positive or negative items and the subject was aware of this fact. In this condition it was again necessary for the subject to detect only one positive or one negative item. Moreover, because the subject could make a decision based on any item in the probe, even more so than in the previous experiment, only a small or absolutely no order effect was predicted. Also, response times were expected to be shorter than in the one-positive case since any one item was decisive. The effects of grouping the memory 


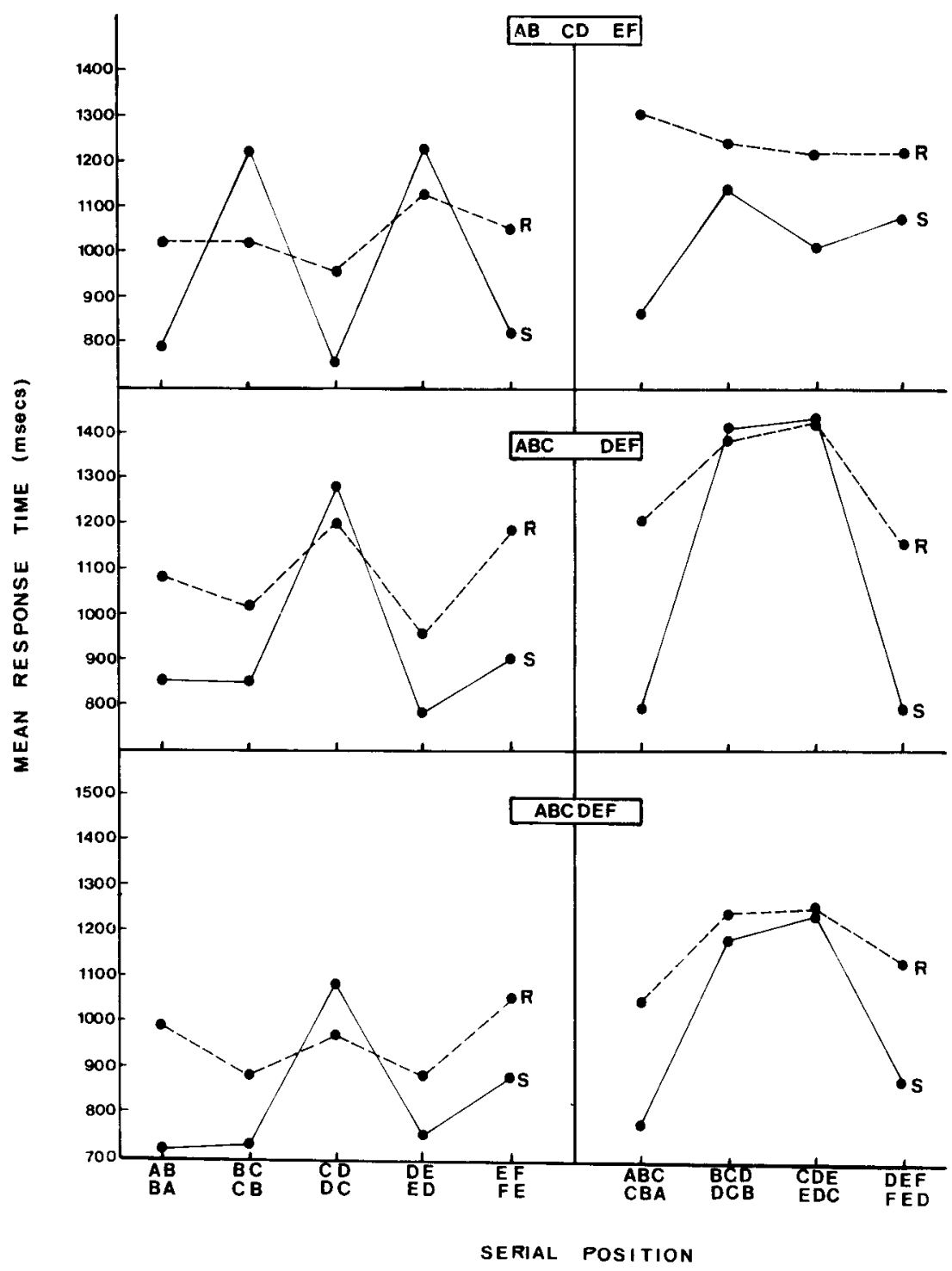

Figure 2. Mean latencies for the six conditions in Experiment 3 of Pike et al. (1985). Memory set groupings appear in the boxes. $\mathbf{S}$ denotes same-order probes; $\mathbf{R}$ denotes reverse-order probes. From "Order Effect in Recognition Latency for Multiple-Item Probes" by A. R. Pike, L. Dalgleish, and K. Jackson, 1985, Journal of Experimental Psychology: Learning, Memory, \& Cognition, 11, Figure 2. Copyright 1985 by the American Psychological Association. Printed with permission.

set in this condition were difficult to predict, so Experiment $2 \mathrm{a}$ with homogeneous probes involved a variety of grouped memory sets. The ungrouped condition was then studied in Experiment $2 b$.

\section{Experiment 2a}

\section{Method}

Subjects. Four undergraduate students were the subjects in this experiment. They were tested individually for five 1.5 -h experimental sessions of 285 trials each, plus a practice session. The subjects completed three of these sessions as a course requirement and were paid for the additional two sessions.

Apparatus. The apparatus for presenting the stimuli and recording the responses were the same as for Experiment 1. There were five memory set size/probe set size conditions, and each was tested separately in different sessions. The conditions were $4 / 2,6 / 2,6 / 3$, $8 / 2$, and $9 / 3$, where the first number refers to memory set size and the second number to probe set size.

A positive probe contained two or three adjacent items from its associated memory set. The letters in the positive probe were either in the same order or in reverse order relative to their memory set positions. A negative probe also contained two or three consonants, but none of the negative probe consonants had been in the associated memory set.

The procedure was essentially the same as for Experiment 1 . Since different-sized memory sets were used in this experiment, each memory set display was presented for a period of time that was the sum of $500 \mathrm{msec}$ per consonant in the memory set. The five experimental conditions were presented in a different random order 


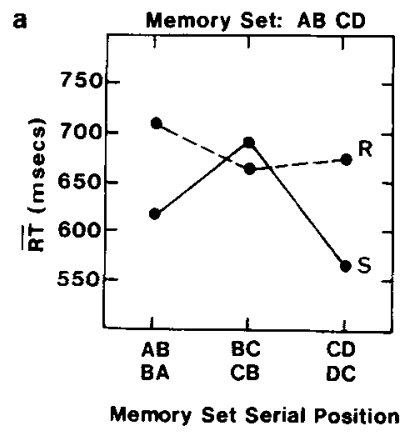

b

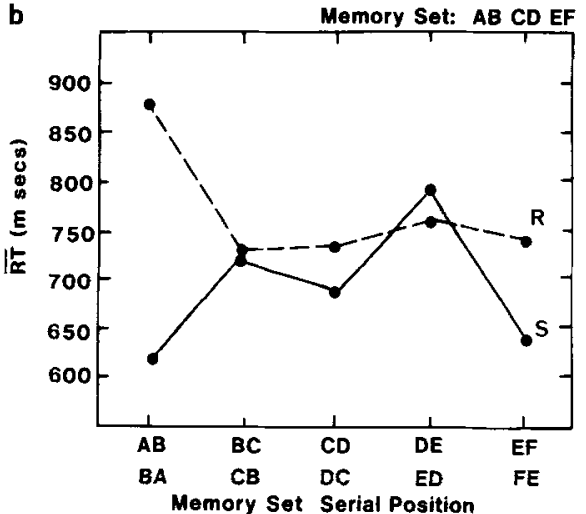

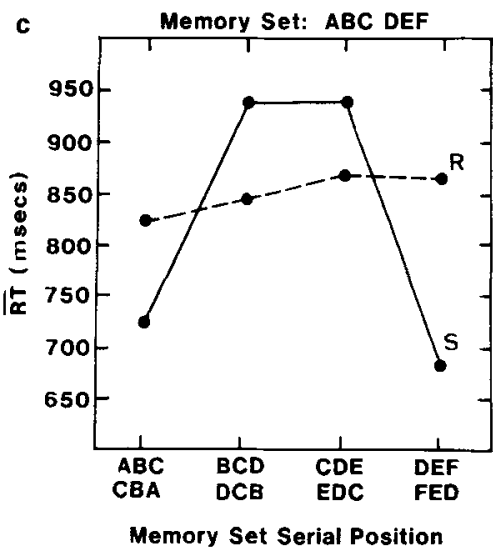

d

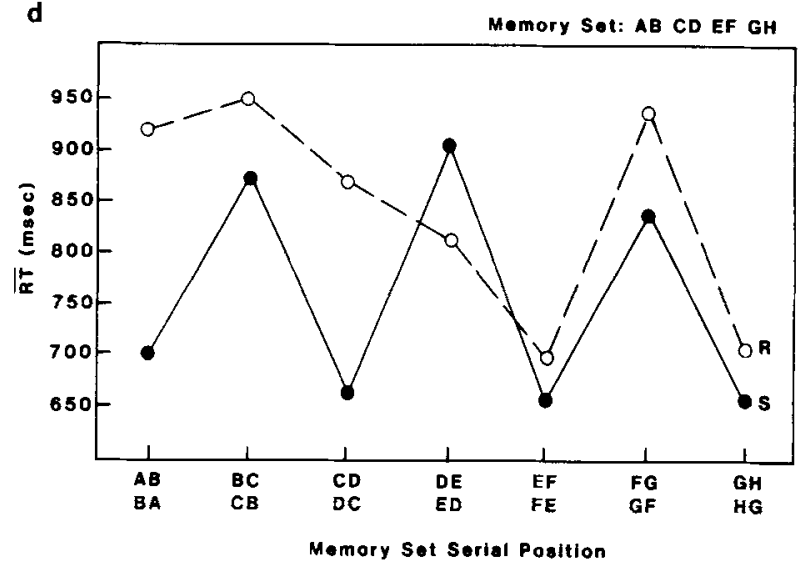

e

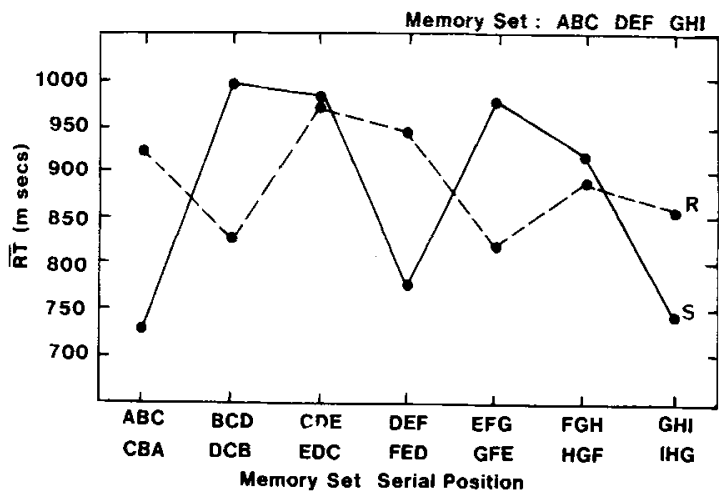

Figure 3. Mean latencies for correct same-order (S) and reverse-order (R) probes in Experiment 2a. Memory set and probe set ar as indicated, and are referred to in the text as $4 / 2$ (a), $6 / 2$ (b), 6/3 (c), 8/2 (d), 9/3 (e).

for each subject. The memory set size was the same as the probe set size, so that set groups were of two or three items. The subjects were informed of the homogeneous nature of the probes.

\section{Results and Discussion}

We are again concerned only with correct positive RTs. A significant order $\times$ serial position interaction was found for four of the experimental conditions. For each of the five memory set size/probe set size conditions, an ANOVA was performed with the two factors of order (same/reverse) and serial position. Results of $F$ tests are as follows for order, serial position, and their interaction, respectively: For $4 / 2, F(1,3)=13.4, M S \mathrm{e}=2,627$, $p<.05$; n.s.; $F(2,6)=5.76, M S \mathrm{e}=888, p<.05$. For $6 / 2$, n.s.; n.s.; $F(4,12)=9.63, M S \mathrm{e}=9,482, p<.01$. For $6 / 3$, n.s.; $F(3,9)=7.8, M S \mathrm{e}=8,759, p<.01$; $F(3,9)=9.69, M S \mathrm{e}=4,340, p<.01$. For $8 / 2$, n.s.; $F(6,18)=4.38, M S \mathrm{e}=15,395, p<.01 ;$ n.s. $[F(6,18)$ $=2.4]$. For $9 / 3$, n.s.; $F(6,18)=3.4, M S \mathrm{e}=9,871$, $p<.05 ; F(6,18)=4.73, M S e=8,393, p<.01$.

Error rates were $6.1 \%$ for same-order probes and $12.6 \%$ for reverse-order probes.

The extent of order and serial position effects was unexpected, because the homogeneous probe condition was made very clear to subjects. A subject's responses to positive probes were markedly influenced by the grouping conditions of the memory set, so much so that the overal order effect was virtually obliterated. The marked effec of the memory set grouping on latency is most clearly illustrated in Figure 3c, which shows a significant orde1 $x$ serial position interaction. These data are similar tc some of the data obtained by Walker (1972) and suppor his suggestions that subjects use features of the presentec stimulus structure to enable encoding and storage of items.

Apparently, the order effect is not present in four of the five conditions because of the fact that substantial reverse-order effects occur when probes are between groups, that is when they contain items from two groups in the memory set. This is clearly shown in Figures $3 \mathrm{t}$ and $3 \mathrm{c}$, where the general similarity of the data to the earlier data for two groups of 3 with probe set size 3 and three groups of 2 with probe set size 2 may be noted. The breakdown of the order effect for positive between-grour probes is again strong support for our assumption that the presence of grouping in the memory set is responsible for the order effect. However, although we have previously suggested that the order effect increases with an increase of grouping in the data, we now have to modify that suggestion because of the possibility of reverse-order effects. The presence of reverse-order effects indicates that grouping effects may be quite substantial at the same time that the overall order effect is quite small. 
Important features of the subjective grouping-order effect relationship are evident in Figure 3. It appears that the first item in the probe is particularly decisive. If this item is also the first item in a group, then a faster response time ensues. If a whole group is represented in the sameorder probe, then it is responded to fastest. On the other hand, between-group probes do not match subjective groups, and hence show no positive order effect. Reverseorder effects with between-group probes are discussed later.

As expected, mean latencies are lower than in Experiment 1 . This suggests that the single-item comparisons are proceeding in parallel, with only one positive comparison necessary for a response, although this has to be qualified by the apparent faster response times for first items. As to the explanation of the order and serial position effects, if single-item comparisons do not occur in all trials, then the order effects must be quite strong on those trials in which holistic comparisons take place in order to account for the overall data. We prefer the alternative interpretation in which both processing strategies take place simultaneously, but in which the variation in latency for the holistic match process is considerably greater than that for the single-item process, so that on many trials a fast holistic response occurs. It is also possible that it is the combined matching strength of these processes that produces the short response times; this strength is substantial in the homogeneous probe condition.

We next attempted to discover how important the presence of actual grouping in the memory sets is.

\section{Experiment 2b}

\section{Method}

Subjects. Four undergraduate students were paid at a standard rate for participation.

Procedure. In this study the memory set and probe set combinations were the same as in the previous experiment, but memory sets were presented without the imposition of any grouping and the combinations were presented in a trial-by-trial random order. There were five sessions of $\mathbf{2 0 0}$ trials each. The items were presented as part of a simultaneous horizontal array of $4,6,8$, or 9 consonants, with no spacings between any of the letters.

\section{Results and Discussion}

There were no significant interactions between order and serial position, but there were some order effects. A separate two-way ANOVA was carried out on each of the five conditions; the two factors were order and serial position. Mean RT for same-order probes was significantly shorter than that for reverse-order probes $(.743 \mathrm{sec}$ vs. $.798 \mathrm{sec}$ ) for the $4 / 2$ condition $[F(1,3)=10.68, M S \mathrm{e}=$ $20,904, p<.05]$. In addition, for the same experimental condition, there was a significant serial position effect $[F(2,6)=8.9, M S e=5,017, p<.02]$. For the $6 / 2$ condition, mean RT for same-order probes was significantly shorter than that for reverse-order probes $(.823 \mathrm{sec}$ vs. $.884 \mathrm{sec})[F(1,3)=15.17, M S \mathrm{e}=2,020, p<.05]$. Error rates were $7.2 \%$ for same-order and $9.8 \%$ for reverse-order probes.
The absence of interactions indicated that there are not many subjective grouping effects. The subjects apparently based their decisions largely upon single items, and, as a consequence, the overall order effect is very drastically reduced, in line with our prediction. However, because of the range of memory set sizes, it may be that the subjects were deterred from grouping due to the lack of a consistent relationship between memory set size and probe size. Subjects may require a constant memory set size for many trials before subjective item grouping can occur, especially in the homogeneous probe condition in which it is not necessary to test every item in the probe set. This seems to indicate that the use of subjective grouping is influenced by the consistency of memory set size and probe set size. Therefore, as a further test of the grouping/order effect hypothesis, we used a constant ungrouped memory set size of 6 , which seems to be the best condition for subjective grouping to occur.

\section{Experiment $2 c$}

\section{Method}

Subjects. Five undergraduate students were individually tested for six 1.5-h experimental sessions of 285 trials each, after one practice session. They completed $3 \mathrm{~h}$ of testing as a course requirement, and were paid at a standard rate for the remaining time.

Apparatus and Procedure. The apparatus and general procedure were the same as for the previous experiments.

Stimuli. Six separate sets of 240 test trials were developed, one for each session of testing. Within a trial, each memory set was prepared by randomly selecting, without replacement, 6 consonants from the full set of 21 consonants. A probe set was prepared for each memory set. A positive probe contained 1,2,3, or 4 adjacent items from its associated memory set. For positive probes containing more than 1 consonant, the letters were in either the same order or in the reverse order relative to their memory set positions. A negative probe also contained $1,2,3$, or 4 consonants, but none of the negative probe consonants had been in the associated memory set. Probe size varied randomly throughout a session.

\section{Results and Discussion}

Same-order probes were responded to more quickly than reverse-order probes for all probe set sizes, and serial position effects were prominent. The difference in mean RT between same-order and reverse-order probes ranged from $65 \mathrm{msec}$ for probe set size 2 to $50 \mathrm{msec}$ for probe set size 4 . The differences were all statistically significant as follows: for probe set size $2, F(1,4)=15.89, M S e$ $=3,220, p<.01$; for probe set size $3, F(1,4)=13.7$, $M S \mathrm{e}=2,628, p<.02$; for probe set size $4, F(1,4)=$ $23.62, M S e=794, p<.01$. Error rates were $5.4 \%$ for same-order and $13.4 \%$ for reverse-order probes.

There was a significant serial position effect for probes with one item $[F(5,20)=2.87, M S \mathrm{e}=1,210, p<.01]$ and significant serial position $\times$ order interactions for probes with 2, 3, and 4 items as follows: for probe set size 2, $F(4,16)=3.98, M S e=4,972, p<.02$; for probe set size $3, F(3,12)=3.43, M S e=4,231, p<.05$; for probe set size $4, F(2,8)=4.32, M S e=1,174$, $p<.05$. Overall mean RTs for positive probe sizes 1 , 2,3 , and 4 were $619,629,628$, and $638 \mathrm{msec}$, respec- 

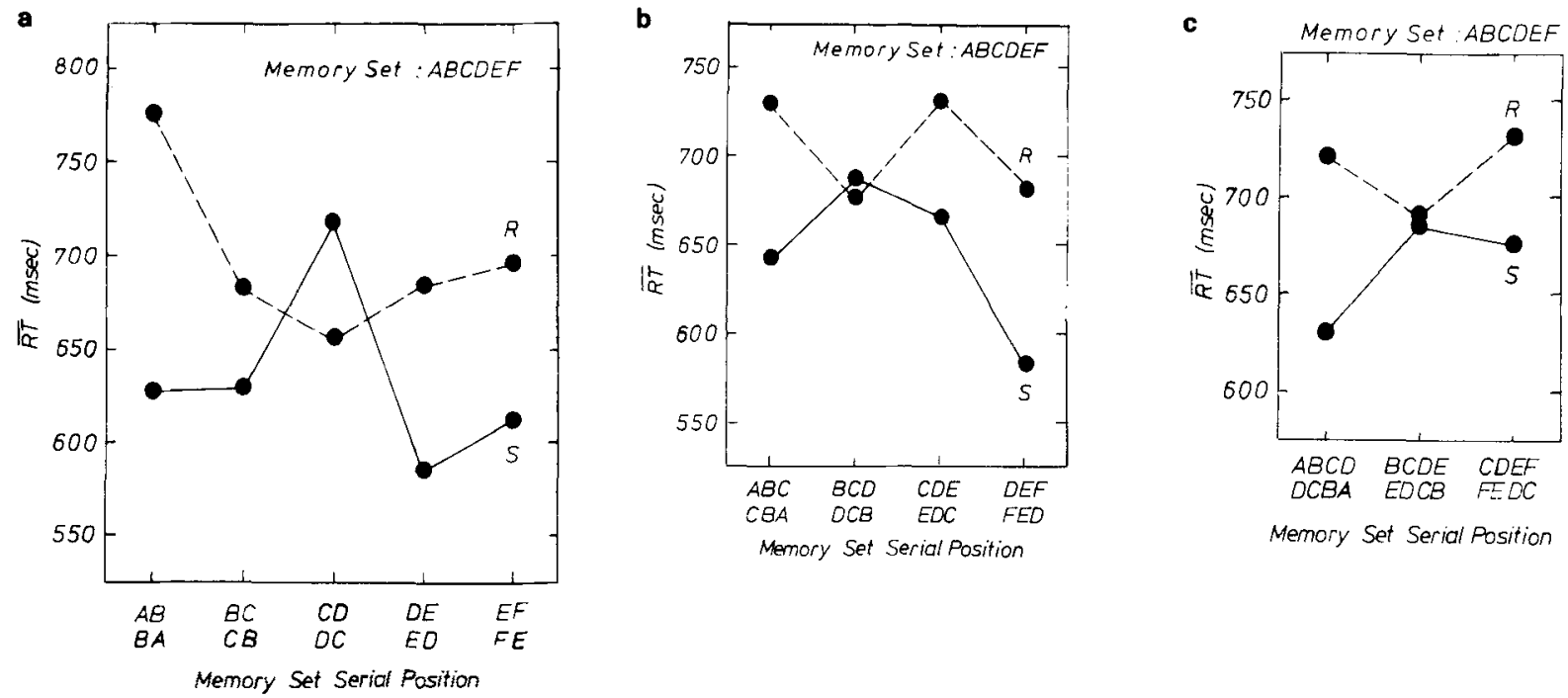

Figure 4. Mean latencies for correct same-order (S) and reverse-order (R) probes in Experiment 2c. Memory set and probe set are as indicated, and are referred to in the text as $6 / 2$ (a), 6/3 (b), 6/4 (c).

tively, and for negative probes were $690,602,677$, and $724 \mathrm{msec}$, respectively.

The use of a constant memory set size has produced strong order effects and subjective grouping in the homogeneous probe condition. The resemblance of the condition shown in Figure $4 \mathrm{a}$ to the similar condition shown in Figures 1 and 2 (two groups of three, probe size 2) is striking. There is also some resemblance to the corresponding condition with two groups of three, probe size 3 . Figures $4 a, 4 b$, and $4 c$ show that the order effect is well established for probe sizes 2,3 , and 4 and that there is no increase in the effect with probe size. Subjects appear, especially from the evidence presented in Figure $4 \mathrm{a}$, to have subjectively grouped the memory set into two three-item groups, ABC and DEF. Thus, sameorder probes $A B, B C, D E$, and $E F$ have low mean RTs, whereas the same-order between-group probe $\mathrm{CD}$ has a much longer RT. In addition, the order effect is greatest for the $A B / B A$ and $D E / E D$ probe pairs. The situation is similar for probe set sizes 3 and 4 , the order effect being greatest for within-group probes and least for betweengroup probes. Overall, our remarks made for Experiment 2 a concerning the order effect and grouping with homogeneous probes apply equally well to the ungrouped constant set size condition.

The data of Experiments $2 \mathrm{a}, 2 \mathrm{~b}$, and $2 \mathrm{c}$ indicate that subjective grouping is an important part of the processing strategy even in those memory tasks in which any single probe item can determine the response. The data also indicate that single-item processing may often occur, especially when subjects are presented with varying memory set sizes. This is indicated by the reduction of serial position effects in Experiment 2b. Thus it appears worthwhile to develop a detailed model, based on the formation of subjective groups and the holistic comparisons of probes with those subjective groups to account for the order and serial position effects. However, it also seems desirable to incorporate single-item matching into the model if it is possible to do so. Our present interpretation of the combined use of holistic and single-item processing may then be justified.

\section{EXPERIMENT 3}

In the previous experiments we used only probes with items that were adjacent in the memory set. It is important to obtain data from probes containing items that are not adjacent, because these probes will always tend to range across subjectively formed groups, and hence predicted latencies from them should realize strong tests of any model. We therefore looked at the latencies and order effects for different patterns of nonadjacency as compared with those for probes with adjacent items. To ensure strong order effects, at least for the latter probe type, we used the all-positive decision rule in this experiment.

\section{Method}

Subjects. Fifteen undergradaute students, who obtained course credit, served as subjects.

Stimuli. Memory set items were randomly sampled from the 21 consonants, without replacement. Positive test probes were constructed into six types, as described in Table 1, according to their pattern of nonadjacency (or spaces between the items of the probe). Each probe subtype was equally likely, and the proportion of positive probes (same and reverse orders) was 0.5 , with negative probes containing one, two, or three negative items in equal proportion.

Procedure. The subjects were tested individually in a soundattenuating chamber. Memory sets and probes were presented on a computer terminal, with both target and probe items appearing as a continuous string in the middle of the screen. Responses were recorded by two push-button microswitches mounted on a metal response board held by the subject, who rested each thumb on a response button. A red light located on the response box served as a trial warning signal. Presentation of stimuli and recording of RT was controlled by computer. Three different experimental sequences of 240 trials, randomized over conditions, were constructed and allocated to the subjects randomly. 
Each trial consisted of a 3-sec red warning signal followed by the simultaneous presentation of the six-letter target set. After $3 \mathrm{sec}$ the display was terminated and the three-letter test probe was displayed. The test probe was either response terminated or automatically terminated after $4 \mathrm{sec}$. A delay of $2 \mathrm{sec}$ occurred before the beginning of the next trial. The subjects were instructed to respond as quickly and accurately as possible. The all-positive decision rule was described to the subject as requiring a "yes" response only if all members of the test probe were members of the target set; otherwise a "no" response was appropriate (i.e., if two, one, or none of the test probe items were members of the target set). The experimental session began with practice on 55 trials to familiar ize the subject with the equipment and decision rule. There were 240 experimental trials, and 5-min rests were given after every 80 trials.

\section{Results and Discussion}

Order effects were substantial for the six probe types and were found to vary over probe type. Mean RTs for same-order and reverse-order probes are given in Table 1 . A two-way ANOVA was performed on these data, and the interaction between order and probe type was significant $[F(5,70)=6.54, M S e=12,360, p<.01]$. There was a main effect for order $[F(1,14)=34.8, M S e=$ $157,859, p<.01]$ and for probe type $[F(5,70)=5.11$, $M S e=67,079, p<.01]$, with the order effect increasing from probe types 1 and 2 to probe types 5 and 6 . We discuss and compare the pattern of order effects in these data and make predictions when we outline the theoretical model.

Response latencies were larger in this experiment than in the one-positive condition and much larger than in the homogeneous probe condition. We feel that the grouping and holistic comparison strategy is predominant in this condition due to the necessity for all probe items to be positive. If the single-item comparisons are being used, then each item has to be confirmed as positive; even with a parallel matching process, this could take a time that is, on the average, longer than the majority of holistic comparison times. As stated earlier, the data of our experiments may indicate that the holistic comparison times have a larger variance than the single-item response times. However, in the all-positive case this is not necessary as an explanation for the predominance of holistic processing.

\section{GENERAL DISCUSSION}

Experiments 1 and 2 demonstrated that order and serial position effects occur when single items are sufficient to determine the response. One explanation is that subjective grouping nevertheless occurs in these situations and that holistic comparisons of subjective groups with probes do take place. The extent of single-item processing varies: It is clearly strong in the one-positive condition, but possibly only weak in the homogeneous probe condition, the latter being an unexpected finding. Experiment 3 has shown the prevalence of the order effect for most probe item patterns and yielded data additional to the serial position effect that must be accounted for by any model. In the light of these data, we considered some theoretical explanations for the order effect and the variety of serial position effects.

The first explanation we considered is an associative one in which latency variation is brought about by variation in the associative strength between items and which assumes that such strength is strongest for adjacent items. This kind of model has been successfully used by Shiffrin and Cook (1978) to account for recall of five-item memory sets. For our work, the notion of directional auditory trace is also relevant to such an interpretation (e.g., Drewnowski, 1980), especially since in our earlier work we found evidence for auditory processing. The main difficulty with an associative explanation as far as our data is concerned is in accounting for the reverse-order effects for between-group probes, and for the results of Experiment 3 in which larger order effects were found when probe items were not adjacent in the memory set. Directionality in association between items needs to be considered, but the experimental evidence for this is only weak (see Murdock, 1974), and directionality cannot explain the reverse-order effects. Thus we conclude that although associations between items may be playing a part, they are not critical in our data.

For all three response conditions used in the present study, it is possible to predict an order effect if subjects adopt a serial self-terminating scanning strategy for item matching, due to the order in which items occur in sameorder and reverse-order probes. However, the order effect should then double in magnitude as probe set size increases from 2 to 3 and so on. This prediction was not verified in the data of Pike et al. (1985) for all-positive responding, and does not appear to be the case in the present data. Given subjective grouping, it is possible to obtain reverse-order effects with a serial search model by assuming that speed of comparison is a function of the correspondence of position of items in the probe set and in the memory set. However, the serial position effects predicted by this modified search model are markedly nonsymmetrical due to the serial scan process, whereas most of our data indicate an underlying symmetry.

Two hypotheses that are strong contenders to account for order effect data are position sensitivity and distribution over position. The first of these was proposed by Angiolillo-Bent and Rips (1982), who used the all-positive task with three-item memory sets and probes containing items in any order. Their hypothesis was supported by Proctor and Healy $(1985,1987)$. The second hypothesis was proposed by Ratcliff (1981), who used five-item memory and probe sets and required correct order for a positive response. Proctor and Healy $(1985,1987)$ used both tasks with a variety of conditions.

To simplify the discussion of these two hypotheses, consider a single trial with a three-item memory set $\mathrm{ABC}$ and the probe ACB. According to the position-sensitivity hypothesis, all three letters in the probe gain matching strength. Item $A$ is at a maximum since it is in the correct position, but items $B$ and $C$ gain less strength because they are out of position; in general, distance from correct position determines, inversely, matching strength. It 
Table 1

The Six Probe Types of Experiment 3 and Their Mean Response Times (RTs) for Same and Reverse Orders

\begin{tabular}{clcccr}
\hline $\begin{array}{c}\text { Probe } \\
\text { Type }\end{array}$ & \multicolumn{1}{c}{$\begin{array}{c}\text { Probe } \\
\text { (Same Order Only) }\end{array}$} & $\begin{array}{c}\text { Same Order } \\
\text { RT }\end{array}$ & \% Error & $\begin{array}{c}\text { Reverse Order } \\
\text { RT }\end{array}$ & \% Error \\
\hline 1 & ABC, BCD, CDE, DEF & 1204 & 5.6 & 1370 & 14.0 \\
2 & ABD, ACD, BCE, BDE, CDF, CEF & 1335 & 7.5 & 1508 & 10.0 \\
3 & ACE, BDF & 1348 & 5.0 & 1720 & 13.2 \\
4 & ABE, ADE, BCF, BEF & 1310 & 4.2 & 1688 & 18.5 \\
5 & ACF, ADF & 1318 & 4.6 & 1814 & 16.2 \\
6 & ABF, AEF & 1156 & 4.3 & 1617 & 17.4 \\
\hline
\end{tabular}

Note-Each probe type has a different pattern of nonadjacency relative to the memory set ABCDEF as follows: 1 , adjacent items; $2, \mathrm{XX}-\mathrm{X}$ or X-XX; $3, \mathrm{X}-\mathrm{X}-\mathrm{X} ; 4, \mathrm{XX}-\mathrm{X}$ or X-XX; $5, \mathrm{X}-\mathrm{X}-\mathrm{X}$ or X--X-X; $6, \mathrm{XX}--\mathrm{X}$ or $X$---XX; where $X$ denotes different memory set items in same or reverse order.

is clear that the position-sensitivity process is not one of matching the probe configuration with the memory set configuration; rather, it is some kind of parallel process dealing with single items (see Angiolillo-Bent \& Rips, 1982). According to the distribution-over-position hypothesis, it is also the case that all items in the probe ACB gain matching strength, but due to the hypothetical distribution that each memory item has over the positions of the memory set. It is as if each memory set item spreads itself out over the possible positions, with the maximum strength of occurrence being at the actual position of the item. Ratcliff (1981) attempted to account for position errors in memory but did not specify the reason for the distributions. He admitted to an affinity of his hypothesis with the perturbation model of Estes (1972), but denied that perturbation of items brings about the distributions. That is, he did not say that the hypothesis defines a probabilistic distribution of each item position over many trials due to perturbation and thus bringing about a distribution-over-position effect over those trials. Thus the Ratcliff (1981) hypothesis is ambiguous as to the origin of the process, other than that given by its mathematical definition.

Ratcliff (1981) pointed out that the positive probe latencies in the Angiolillo-Bent and Rips (1982) data are predicted better by the distribution-over-position hypothesis and that the position-sensitivity hypothesis fails to predict his "switch" versus "double replace"' data. The position-sensitivity process may certainly have difficulty in accounting for all of our data, and seems to predict much larger order effects as set size increases. Until the process is spelled out in greater detail for six-item memory sets and subjective grouping, which may be difficult to do, we cannot be more definite.

There are, however, three points that can be made concerning the intensive studies of Proctor and Healy (1985, 1987). First, one reason for their conclusion in favor of the position-sensitivity hypothesis is because of their assumption that the similarity in the overall pattern of the data for their different experimental conditions implies identical processing. However, it is conceivable that broad patterns in the data follow as a result of task similarity. Thus the correspondence of item positions in the memory and probe sets must surely be an important determiner of response time, so that whatever the experimental con- ditions, there should be a strong relationship between probe disorder and probe latency. Proctor and Healy $(1985,1987)$ conceded that the final response seems to be based on a pooled output, and their argument for this is very similar to that made by Ratcliff (1981) for his distribution-over-position hypothesis. Thus if they were to revise their assumption concerning identity of process, they could more easily accept the latter hypothesis as being as good an explanation of their data as that of position sensitivity. Second, their conclusion was guided by the fact that the strong displacement effects they found in the data for four-item memory sets accorded well with the position-sensitivity appraoch rather than other permutational hypotheses. However, the general notion of displacement effects can just as well be utilized by the distribution-over-position approach if the Ratcliff hypothesis is extended in terms of the model to be described below. In our model, multiple displacement effects in memory are the main cause of variations in matching strength of probes, and hence in reaction time. Third, there is the apparent prediction from the positionsensitivity hypothesis that the order effect should increase as the memory set increases from three to four items, at least in the order-irrelevant all-positive task, because of the increase of displacement from four to eight. This is not borne out in Proctor and Healy's data (for the item task, same and reversed orders, Experiments 1 and 2, being about $400 \mathrm{msec}$ in both cases).

In our previous paper (Pike et al., 1985), we briefly suggested a possible explanation for the occurrence of distributions over position. We now emphasize it again and spell out the details in the Appendix. It is based on the notion of multiple representations in memory and states that on any one trial the memory set is represented in memory several, and perhaps very many, times. Some of these representations are incorrect in item order or contain negative items, so there are some correct representations and some incorrect ones. It is this variation of representation that can easily bring about a distributionover-position situation if it is assumed that the probe representation is matched globally with all representations of the memory set. That is, the matching strength is aggregated over all matches of the probe with each separate representation. Furthermore, due to the presence of the incorrect representations, a distribution over positions oc- 
curs for each item on each trial. In our case it is each subjective group that has a multiple representation so that there is a distribution over the positions of the subjective group for each item contained by that group.

Given the above multiple representation and global matching assumptions, together with subjective grouping, it is shown in the Appendix how to account for our serial position data and the latency pattern found in Experiment 3. We take the ordering of the frequencies of representation of the three-item groups and their incorrect permutations to correspond inversely with the ordering of the mean latencies for those permutations, presented as probes, in the results of Angiolillo-Bent and Rips (1982). This applies for both subjective groups $A B C$ and $\mathrm{DEF}$, given the memory set $\mathrm{ABCDEF}$, and similarly for the other memory sets used in our experiments. These assumptions turn out to be sufficient to predict the form of the serial position effects we obtained in Experiments 1 and 2 and in the earlier work. The assumptions are restrictive insofar as serial position effects greatly different from these would have to be explained by implausible representation patterns in memory, such as where the correct representation $\mathrm{ABC}$ was not the more frequent, on the average. The model is also testable with the data of Experiment 3, where the ratio of order effect magnitudes for the six probe types (1-6) is about $1: 1.06: 2.2: 2.3: 3: 2.8$, and the predicted ratios are shown in the Appendix to be $1: 1: 2: 2: 3: 3$. The product-moment correlation between the 12 observed mean latencies is 0.95 . The predicted equality of order effects for probe types 3 and 4 and for types 5 and 6 is very general and provides, therefore, a strong test of the model.

Unfortunately, the model as it stands is difficult to specify in terms of a small set of parameters, since the multiple representation pattern within memory can easily be varied both theoretically and, if the theory were correct, in actual fact. Thus the biggest problem is in stating a simple algorithm that would apply to many different memory and probe set sizes. The model is essentially one that concerns variation in representation in memory and may well be justified in its parameter flexibility. However, it is shown in the Appendix that quite simple versions of the model are sufficient to give a first approximation to the data. In the absence of a simple account for all of our data, we present the model as an initial contribution toward the explanation of order effects. The more usual conception of single representation in memory leads to simple models and the subsequent easy analysis by search models, but for selected situations. Currently, however, a powerful idea concerning memory systems is proving attractive, and it is one to which the multiple representation model can be made congruent. This is the idea of distributed memory processing (e.g., Anderson, 1973; Hintzman, 1986; Murdock, 1982; Pike, 1984). It is possible to conceive of all representations, as we have defined them, being held in store as one single distributed memory. The probe has then to match only this single memory in the process of recognition. Distributed memories have powerful and interesting ways of modeling various memory processes (e.g., Eich, 1985; Humphreys, Pike, Bain, \& Tehan, 1987), and thus links between order effects found here and those found in more complicated memory events may be found.

\section{REFERENCES}

Anderson, J. A. (1973). A theory for the recognition of items from short memorized lists. Psychological Review, 80, 417-438.

Angiolillo-Bent, J. S., \& RIPS, L. J. (1982). Order information in multiple-element comparison. Journal of Experimental Psychology: Human Perception \& Performance, 8, 392-406.

BaUmgarte, R., De Rosa, D. V. (1973). Information processing in a two-item classification task: Relationship among items in a memorized set. Joumal of Experimental Psychology, 100, 1-7.

DrewnowskI, A. (1980). Attributes and priorities in short-term recall: A new model of memory scan. Joumal of Experimental Psychology: General, 109, 208-250.

EICH, J. M. (1985). Levels of processing, encoding specificity, elaboration and CHARM. Psychological Review, 92, 1-38.

ESTES, W. K. (1972). An associative basis for coding and organization in memory. In A. Melton \& E. Martin (Eds.), Coding processes in human memory (pp. 161-190). Washington, DC: Winston.

HintzMAN, D. L. (1986). Schema abstraction in a multiple-trace memory model. Psychological Review, 93, 411-428.

Humphreys, M. S., Pike, R., BaIn, J. D., \& Tehan, G. (1987). Global matching: A comparison of the SAM, Minerva and Matrix models. Unpublished manuscript.

MuRDock, B. B. (1974). Human memory: Theory and data. Potomac, MD: Erlbaum.

MuRDock, B. B. (1982). A theory for the storage and retrieval of item and associative information. Psychological Review, 89, 609-626.

PIKe, A. R. (1984). Comparison of convolution and matrix distributed memory systems for associative recall and recognition. Psychological Review, 91, 281-294.

Pike, A. R., Dalgleish, L., \& Jakson, K. (1985). Order effect in recognition latency for multiple-item probes. Joumal of Experimental Psychology: Learning, Memory, \& Cognition, 11, 248-261.

Proctor, R. W., Healy, A. F. (1985). Order-relevant and orderirrelevant decison rules in multiletter matching. Joumal of Experimental Psychology: Learning, Memory, \& Cognition, 11, 519-537.

Proctor, R. W., \& Healy, A. F. (1987). Task-specific serial position effects in comparisons of multiletter strings. Perception \& Psychophysics, 42, 180-194.

RATCLFF, R. (1981). A theory of order relations in perceptual matching. Psychological Review, 88, 552-572.

ShIFFrin, R. M., \& CoOK, J. R. (1978). Short-term forgetting of item and order information. Journal of Verbal Learning \& Verbal Behavior, 17, 189-218.

WALKER, E. C. T. (1972). The effect of structure on scanning strategies. Perception \& Psychophysics, 12, 427-429.

\section{APPENDIX}

We first describe a simple case of how a distribution of item strength over position may be generated and the subsequent matching process. Suppose the memory set ABCDEF is presented, and the subjective groups $A B C$ and $D E F$ are retained in a multiple representation pattern of $3 \times \mathrm{ABC}, 1 \times \mathrm{ACB}$, $1 \times \mathrm{CBA}, 3 \times \mathrm{DEF}, 1 \times \mathrm{EDF}$, and $1 \times \mathrm{FED}$. Then the discrete distributions over the three positions of the two three-item groups are $4,0,1$ for $A ; 0,4,1$ for $B ; 1,1,3$ for $C ; 3,1,1$ for $D ; 1,4,0$ for $E$; and 1,0,4 for $F$ (e.g., the item $A$ occurs four times in position 1 , never in position 2 , and once in position 3). Now consider the matching of the probe $\mathrm{ABC}$ and its reversed order CBA with all representations in memory. The probe $\mathrm{ABC}$ has a total matching strength of 11 ( 4 for $A+4$ for $B+3$ for $C$ ), 
whereas CBA has only $6(1+4+1)$. For the between-group probes BCD and DCB, the strengths are $2(0+1+1)$ and 5 $(3+1+1)$, respectively. The strengths vary similarly for the remaining probes.

A simple translation of strength into theoretical latency for each probe is Latency $=1+a-S$, where $a$ is the maximum strength for that set of probes ( 11 in this case) and $S$ is the total strength. Then the latencies for $\mathrm{ABC}$ and $\mathrm{DEF}$ are equal to 1 , for $\mathrm{CBA}$ and $\mathrm{FED}$ are equal to 6 , for $\mathrm{BCD}$ and $\mathrm{CDE}$ are equal to 10, and for DCB and EDC are equal to 7 . These predicted latencies are plotted in Figure Ala, where a marked resemblance to the data of Figure $3 \mathrm{c}$ can be seen. A modified representation of the subgroups gives a latency pattern very near to the $A B C$ DEF and ABCDEF conditions for three-item probes in Figure 2.

Given the same pattern of multiple representation and latency transfer function, consider the two-item probes $\mathrm{AB}, \mathrm{BC}$, and so forth. In this case we make the arbitrary assumption that the probe is matched with the first two and last items in each subjective group of three items, and the matching strength is taken to be the maximum of these two (taking the sum leads to similar results). Then the matching strengths for $\mathrm{AB}$ and $\mathrm{EF}$ are 8, for $\mathrm{BC}$ and $\mathrm{DE}$ are 7 , and for $\mathrm{CD}$ is 2 , and matching strengths for their reverse orders are 5, 5, and 4, respectively. These predicted latencies are plotted in Figure Alb where a marked resemblance can be seen to the latency pattern for the $A B C D E F$ and ABCDEF conditions for two-item probes in Figure 2.

For memory sets presented as three two-item groups, the pattern of representation may be quite different, which is not surprising since two-item groups are perceptually very different from three-item groups. From the representation pattern necessary to fit this data, the first and last items in the first and third groups seem to obtain most of the memory strength, whereas the item strength is equally divided for the middle group. That is, a very strong single-item representation for $\mathrm{A}$ and $\mathrm{F}$ is required if we are to reproduce the latency pattern for this condition. One such representation is $2 \times \mathrm{AB}, 1 \times \mathrm{BA}$, and $6 \times \mathrm{AA}$ (the single-item representation of $A) ; 5 \times C D$ and $4 \times D C ; 2 \times E F$, $1 \times \mathrm{FE}$, and $6 \times \mathrm{FF}$. There may be, of course, other representations that produce the same result, given in Figure Alc. This latency pattern is seen to be similar to the $\mathrm{AB} C \mathrm{CD}$ EF condition with two-item probes in Figure 2. Using the same representation pattern and matching process as described above for probe size 2 and subgroup size 3 , the predicted pattern for the $A B C D$ EF condition with probe size 3 is simply a pair of parallel lines (see Figure 2).

Thus the model makes quite reasonable predictions in these simple cases. To obtain closer fits of the model to the data, more complex multiple representation patterns of the memory sets can be specified. To keep some theoretical restraint on these patterns, we may utilize the results of Angiolillo-Bent and Rips (1982) with three-item memory sets as a guideline. In that study, the ascending order of mean latency for the probes was $A B C$, $\mathrm{ACB}, \mathrm{BAC}, \mathrm{BCA}, \mathrm{CAB}$, and $\mathrm{CBA}$, but with little difference in the mean latencies, and hence the ordering of the last three. That is, we assume that given the memory set $A B C$, the representation $\mathrm{ABC}$ is in the majority, that $\mathrm{ACB}$ is usually next in frequency of representation, and so on. Keeping within these guidelines, it is also reasonable to suppose that some representations are incomplete or contain nonmemory set items (e.g., $\mathrm{AB} x$ or $A B Z$, respectively). Given these assumptions it is always possible to generate predictions very close to the data. The data represented in Figure 3a is easily fitted by the model, and that of Figure $3 b$ is clearly similar to the same condition in Figures 1 and 2, fitted as above. The data of Figure $4 a$ can be fitted if arbitrary assumptions about the matching process, similar to that mentioned above, are made for this case. Figure $4 \mathrm{~b}$ also
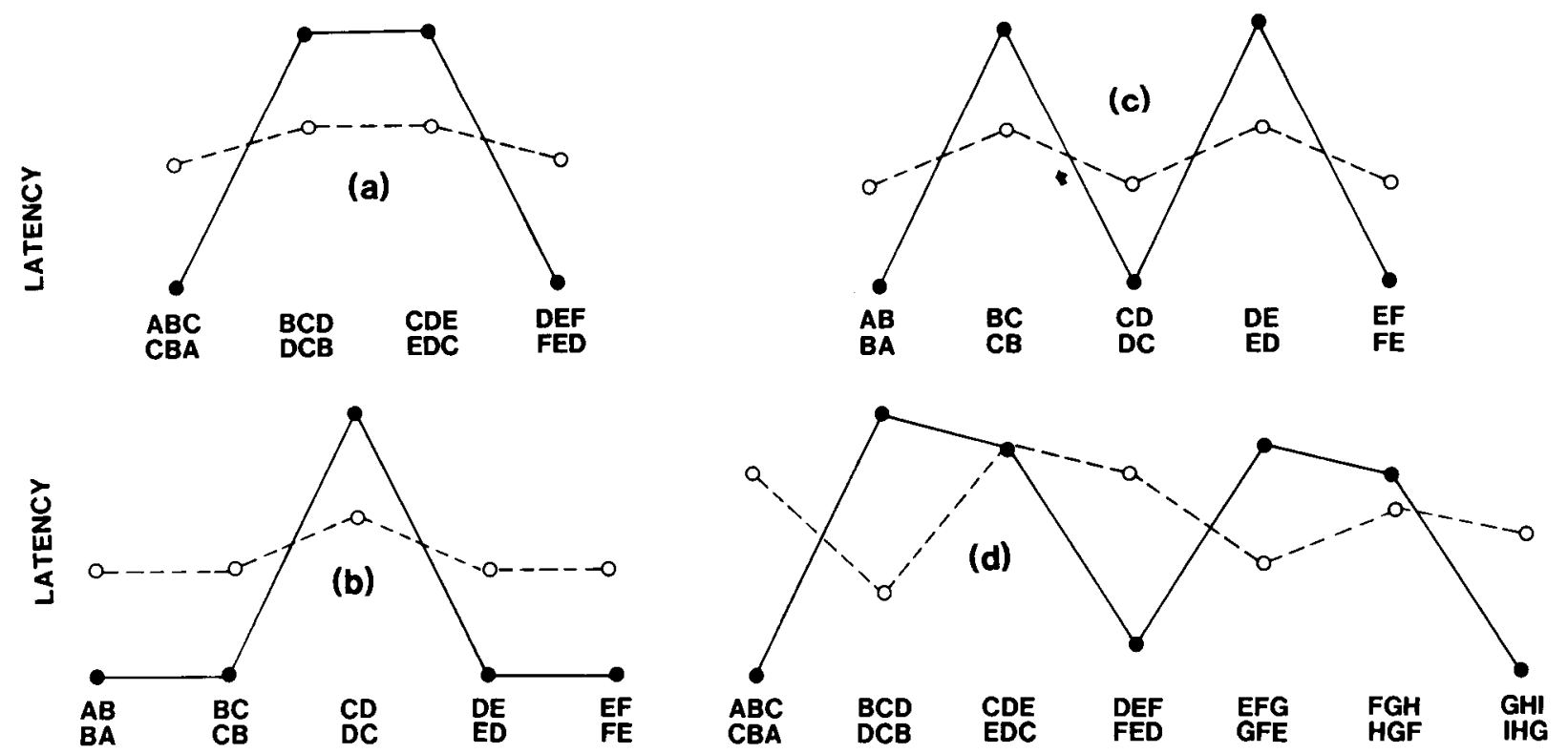

Figure A1. The theoretical serial position latencies for a six-item memory set grouped into ABC and DEF are shown in (a) and (b), respectively, with probes as indicated. Latencies for a memory set grouped into AB,CD,EF are shown in (c). Nine-item memory set (d) grouped into ABC, DEF, GHI, to fit the data of Figure 3d. Dashed lines show reverse-order probes. 
resembles the earlier results, but Figure $4 \mathrm{c}$ has only a tenuous resemblance to that condition in Figures 1 and 2, and we have not attempted to fit those data. The most complicated serial position data is that depicted in Figure 3e for the memory set $A B C$ DEFGHI. Assuming subjective grouping into $\mathrm{ABC}, \mathrm{DEF}$, and GHI, it is not too difficult to construct a pattern of representation along the above lines, which generates a prediction very close to the data, as in Figure A1.

Single-item processing may be incorporated into the model as described above by supposing that items may be represented as a two- or three-item group (AA, $\mathrm{AAA}, \mathrm{BBB}$, etc.). If the situation demands, such as in the one-positive or in the homogeneous probe condition, then all probe items may be thus represented and matched in memory. If processing of this kind is faster than holistic probe processing, it could account for the faster response times for homogeneous probes.

Turning to the data of Experiment 3, the aim is to fit the model to the comparative order effects for the six different probe types. In fact, it is possible to obtain a rough correspondence with the data even when only the subgroups $\mathrm{ABC}$ and DEF are held in memory. Then the distributions over position are simply $1,0,0$ for $A$ and $D ; 0,1,0$ for $B$ and $E$; and $0,0,1$ for $C$ and $F$. Then to obtain predictions for the different probe types, it is necessary to consider each particular case of these types (e.g., for type 1 the cases are $\mathrm{ABC}, \mathrm{BCD}, \mathrm{CDE}, \mathrm{DEF}$ and their reverse orders) and to use the distributions to calculate the strength for each subtype and hence the average order effect for each of the six probe types, assuming that the cases occur equally often in the experiment. The predicted comparative order effects are then $1,0,2,2,4,4$ for the six types, respectively. This prediction remains approximately the same for many multiple representation patterns. To obtain a more accurate fit to the data of Experiment 3 , a more typical multiple representation pattern is required. We have not attempted to find the very simplest pattern to fit the data, so the pattern below is a good example of a more complex representation of the memory set using the guidelines described above.

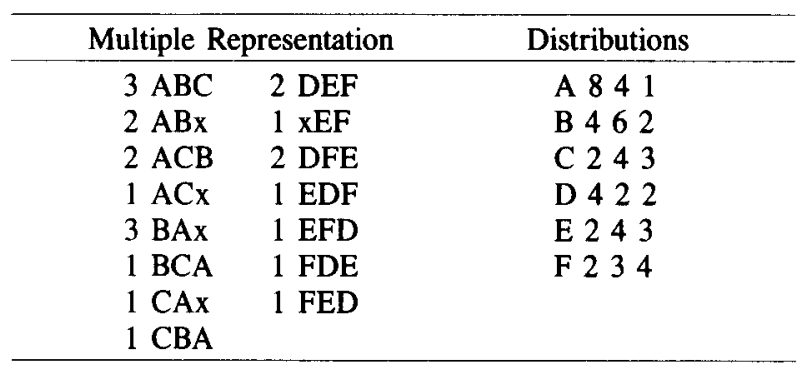

Given the above distributions, the strength for any probe can be calculated as described above. Doing this and making any linear transformation to latency, the order effects for the six probe types of Experiment 3 (types 1 and 6) are of the respective magnitude $1,1,2,2,3,3$, which is in very reasonable agreement with the data. It is, of course, possible to optimize the predictions, although a computer program would be required since any one adjustment to the representation pattern will alter many distribution points. In any case, we would not place much store in such an exercise as it is virtually fitting to "noise." More important for any pattern, a set of 12 strength values is obtained (6 probe types, same and reverse orders), and these 12 strengths may be correlated with the obtained mean latencies. In this case, the product-moment correlation is 0.95 , so the present representation seems sufficiently good in terms of the separate latencies as well as the comparative order effects. In general, for the more plausible representation patterns, the comparative magnitudes of the order effects for the six probe types of Experiment 3 may be said to be $1, x, y, y, z, z$, respectively, where $x$ varied from about 0 to $1, y$ is about 2 , and $z$ is around 3 to 4 . We have obtained other, unpublished, data were $x$ is approximately zero, so this prediction may be a good test of the model.

(Manuscript received March 10, 1986; revision accepted for publication August 28, 1987.) 